\title{
Characterization of intracortical synaptic connections in the mouse anterior cingulate cortex using dual patch clamp recording Long-Jun $\mathrm{Wu}^{1}$, Xiangyao $\mathrm{Li}^{1}$, Tao Chen ${ }^{1}$, Ming Ren ${ }^{1}$ and Min Zhuo ${ }^{* 1,2}$
}

Address: ${ }^{1}$ Department of Physiology, Faculty of Medicine, University of Toronto center for the Study of Pain, University of Toronto, 1 King's College Circle, Toronto, Ontario M5S 1A8, Canada and 2Department of Brain and Cognitive Sciences, Seoul National University, Seoul 151-746, Korea

Email: Long-Jun Wu - longjun.wu@utoronto.ca; Xiangyao Li - leexiangyao@gmail.com; Tao Chen - chtkkl@yahoo.com.cn; Ming Ren - ming.ren@utoronto.ca; Min Zhuo* - min.zhuo@utoronto.ca

* Corresponding author

Published: 15 October 2009

Molecular Brain 2009, 2:32 doi:10.1 186/1756-6606-2-32

This article is available from: http://www.molecularbrain.com/content/2/I/32

(c) 2009 Wu et al; licensee BioMed Central Ltd.

This is an Open Access article distributed under the terms of the Creative Commons Attribution License (http://creativecommons.org/licenses/by/2.0), which permits unrestricted use, distribution, and reproduction in any medium, provided the original work is properly cited.
Received: 20 August 2009

Accepted: 15 October 2009

\begin{abstract}
Summary
The anterior cingulate cortex (ACC) is involved in sensory, cognitive, and executive functions. Studies of synaptic transmission and plasticity in the ACC provide an understanding of basic cellular and molecular mechanisms for brain functions. Previous anatomic studies suggest complex local interactions among neurons within the ACC. However, there is a lack of functional studies of such synaptic connections between ACC neurons. In the present study, we characterized the neuronal connections in the superficial layers (I-III) of the mouse ACC using dual whole-cell patch clamp recording technique. Four types of synaptic connections were observed, which are from a pyramidal neuron to a pyramidal neuron, from a pyramidal neuron to an interneuron, from an interneuron to a pyramidal neuron and from an interneuron to an interneuron. These connections exist among neurons in layer II/III or between neurons located layer I and II/III, respectively. Moreover, reciprocal connections exist in all four types of paired neurons. Our results provide the first key evidence of functional excitatory and inhibitory connections in the ACC.
\end{abstract}

\section{Introduction}

The anterior cingulate cortex (ACC) is the frontal part of the cingulate cortex, which forms a large region around the rostrum of the corpus callosum in the mammalian brain. Studies from both animals and humans consistently demonstrate that the ACC plays a critical role in emotional and attentive responses to internal and external stimulation, such as pain, fear, anxiety, sexual arousal, learning and memory [1-9]. For example, electric or chemical activation of the ACC facilitates the spinal nociceptive tail-flick reflex [10], induces fear memory [11] and aversive learning [12]. Furthermore, peripheral stimulation activates immediate early genes as well as long-term plastic changes in the ACC [13-16]. Therefore, synaptic transmission and plasticity in the ACC are important for ACC-related brain functions.

The ACC is a part of the thalamo-limbic-cortical circuitry where it receives various sensory inputs from the thalamus and sends outputs to motor cortex as well as several subcortical brain regions such as the hippocampus, amygdala and hypothalamus [7,17]. Anatomically, the ACC itself contains several layers including layer I, II, III, V and VI. Layer I contains small local interneurons. However, many projecting fibers from other central nuclei end or pass through layer I. Neurons in layers II-III are mainly pyramidal cells, which receive sensory inputs from the medial thalamus and send projections to deep layers. 
Pyramidal neurons in layer V receive input from layers IIIII as well as the thalamus and project to cortical and subcortical structures $[7,18-20]$. In layers II-VI, there are also many local interneurons. It has been proposed that neurons in the ACC may form local excitatory and inhibitory connections. However, direct evidence for functional connections between pyramidal neurons and/or interneurons within the ACC has not been reported.

Our previous studies indicate that fast excitatory synaptic is mediated by glutamate [14,21] and inhibitory synaptic transmission is mainly mediated by GABA transmission in the ACC [22]. However, the stimulations used in these studies cannot differentiate the exact afferent inputs to recorded neurons, which could be derived from either intra-ACC or from subcortical areas. In the present study, we have examined direct neuronal connections using dual whole-cell patch clamp recording method in ACC slices. To our knowledge, this is the first study using dual recording technique in the ACC region. Our results show different pairs of uni- and bi-directional synaptic connections between pyramidal neurons and/or interneurons in the ACC.

\section{Methods \\ Animals}

All C57BL/6 mice were purchased from Charles River and were maintained on a $12 \mathrm{~h}$ light/dark cycle with food and water provided ad libitum. Experiments were performed on 3-4 weeks old mice. The Animal Studies Committee at the University of Toronto approved all experimental protocols.

\section{Brain slice preparation}

Mice were deeply anesthetized with isoflurane. Coronal brain slices $(300 \mu \mathrm{m})$ containing the ACC were prepared using standard methods $[14,22,23]$. Slices were transferred to a submerged recovery chamber with oxygenated $\left(\begin{array}{llll}95 \% & \mathrm{O}_{2} & \text { and } 5 \% & \mathrm{CO}_{2}\end{array}\right)$ artificial cerebrospinal fluid (ACSF) containing (in mM): $124 \mathrm{NaCl}, 2.5 \mathrm{KCl}, 2 \mathrm{CaCl}_{2}$, $2 \mathrm{MgSO}_{4}, 25 \mathrm{NaHCO}_{3}, 1 \mathrm{NaH}_{2} \mathrm{PO}_{4}, 10$ glucose at room temperature for at least one hour.

\section{Dual whole-cell patch clamp recordings in ACC slices}

After one-hour recovery, slices were placed in a recording chamber on the stage of an Olympus BX51WI microscope (Tokyo, Japan) with infrared DIC optics for visualization of whole-cell patch clamp recordings. Neurons were recorded from layer I or II/III with an Axon 200B amplifier (Molecular devices, CA). Three types of intracellular solutions were used: (1) normal intracellular solution (in $\mathrm{mM}$ ): K-gluconate, $120 ; \mathrm{NaCl}, 5 ; \mathrm{MgCl}_{2} 1$; EGTA, 0.5; MgATP, 2; $\mathrm{Na}_{3} \mathrm{GTP}, 0.1$; HEPES, 10; pH 7.2; 280-300 mOsmol, (2) high $\mathrm{Cl}^{-}$intracellular solution: same as normal intracellular solution except K-gluconate (120 mM) was replaced by $\mathrm{KCl}(60 \mathrm{mM})$ and K-gluconate $(60 \mathrm{mM})$, and (3) low $\mathrm{Cl}$ - intracellular solution: same as normal intracellular solution except K-gluconate $(120 \mathrm{mM})$ was replaced by $\mathrm{Cs}-\mathrm{MeSO}_{3}(120 \mathrm{mM})$. The $\mathrm{Cs}-\mathrm{MeSO}_{3}$ was used to improve the clamp quality. The membrane potential was held at $-70 \mathrm{mV}$ for postsynaptic neurons to record unitary excitatory postsynaptic current (uEPSCs) with the normal intracellular solution, while held at $0 \mathrm{mV}$ to record outward unitary inhibitory postsynaptic currents (uIPSCs) with the low $\mathrm{Cl}^{-}$intracellular solution and at $-70 \mathrm{mV}$ to record inward uIPSCs with the high $\mathrm{Cl}$ - intracellular solution. Access resistance was 15-30 $\mathrm{M} \Omega$ and was monitored throughout the experiment. In dual whole-cell recording, action potentials were elicited by applying brief ( $1 \mathrm{~ms})$ depolarizing current pulses (200 pA) under current clamp configuration or applying brief ( $1 \mathrm{~ms})$ depolarizing voltage pulse (from $-70 \mathrm{mV}$ to $+20 \mathrm{mV}$ ) at $0.1 \mathrm{~Hz}$. The latency of postsynaptic currents was determined by the time difference between the peak of presynaptic spikes and the onset of postsynaptic current. The rise time of postsynaptic currents is the time from $10 \%$ to $90 \%$ of maximal peak current, while the decay time is the time from maximal peak amplitude to $37 \%$ of the peak amplitude. Half-width of postsynaptic currents is the width (duration) at halfmaximal peak amplitude.

\section{Biocytin labeling and confocal imaging}

After recording, brain slices were immediately fixed in $4 \%$ paraformaldehyde in $0.1 \mathrm{M}$ phosphate buffer $(\mathrm{PB}, \mathrm{pH}$ 7.4) for $1 \mathrm{hr}$ at room temperature. Slices were then transferred to $0.01 \mathrm{M}$ phosphate buffer saline (PBS, $\mathrm{pH} 7.4$ ) containing 1\% Triton X-100 (PBS-triton) and stored at $4^{\circ} \mathrm{C}$ for $48 \mathrm{hr}$. After this, sections were rinsed with $3 \%$ hydrogen peroxide in $0.01 \mathrm{M}$ PBS for $30 \mathrm{~min}$. After thoroughly washing with PBS, the tissue was incubated with Fluorescein (DTAF) Streptavidin (016-010-084, 1:200 dilution, Jackson) containing 3\% fish gelatin (Sigma) in PBS-Triton for 4 hours at room temperature. The immunofluorescence-labeled sections were then rinsed in PBS, mounted onto glass slides, air dried, cover-slipped with a mixture of $50 \%(\mathrm{v} / \mathrm{v})$ glycerin and $2.5 \%(\mathrm{w} / \mathrm{v})$ triethylene diamine in $0.01 \mathrm{M}$ PBS, and observed with an confocal microscope (FV-1000; Olympus, Tokyo, Japan) under appropriate filter for DTAF (excitation $492 \mathrm{~nm}$; emission $520 \mathrm{~nm})$.

\section{Data analysis and statistics}

Results were analyzed by t-test and paired t-test where necessary. All data are expressed as mean \pm S.E.M. In all cases, $P<0.05$ was considered statistically significant.

\section{Results}

We proposed that there are complex synaptic connections in the ACC (Figure 1A). In the superficial layers such as I and II/III, pyramidal neurons may send projections to 
A

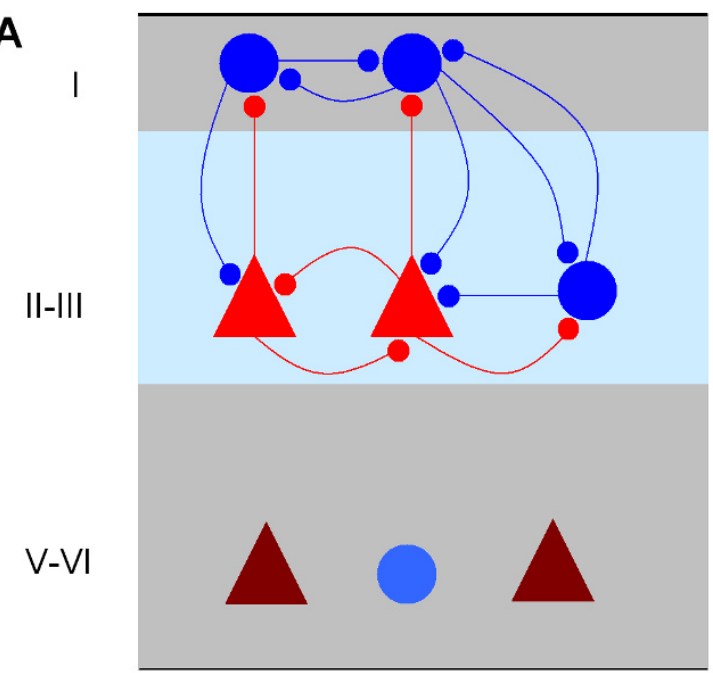

B

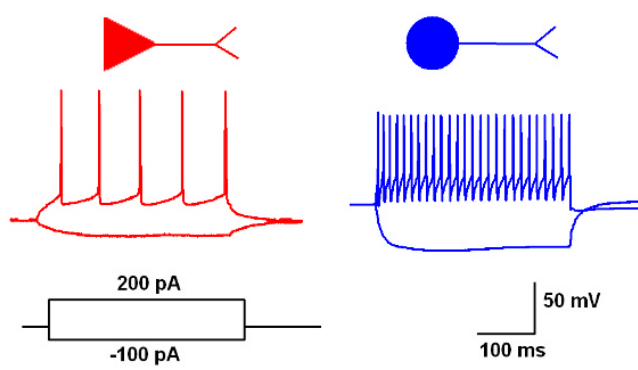

Figure I

Diagram for the proposed synaptic connections in the ACC circuit. (A) A simplified diagram illustrates the possible synaptic connections in the ACC. Pyramidal neurons are indicated as red triangles, while interneurons are blue circle. (B) Typical firing patterns for pyramidal neurons (left) and interneurons (right) after current injection of $200 \mathrm{pA}$ for $400 \mathrm{~ms}$. The resting membrane potentials for the pyramidal neuron and interneuron are $--71.3 \mathrm{mV}$ and $--67.8 \mathrm{mV}$, respectively.

pyramidal neurons and interneurons, interneurons may target pyramidal neurons and interneurons, and reciprocal connections may exist between two pyramidal neurons, a pyramidal neuron and an interneuron, or two interneurons. To test the possibilities, dual recordings were performed in neuronal pairs in layers I and II/III of the ACC. Although we have found that pyramidal neurons and interneurons could also be divided into several subtypes based on firing patterns [24] (Cao et al., unpublished data), we only classify these neurons into pyramidal neuron (Py) or interneuron (In) to simplify the possibilities of neuronal connections. The typical firing patterns for pyramidal neurons and fast-spiking interneurons are shown in Figure $1 \mathrm{~B}$ after current injection (200 pA, $400 \mathrm{~ms})$.

Dual whole-cell patch clamp recordings were performed in the layer I and II/III neurons in the ACC from juvenile, wild-type mice (3-4 week old). This study included recordings from 49 mice and a total of 71 successful synaptic connections were obtained from around 223 pairs of neurons recorded (about 31\% success rate). All pyramidal neurons were recorded from layer II/III while interneurons were recorded from layer I or II/III as specified in the results. Pyramidal neurons and interneurons were distinguished based on their morphology (Figure 5), membrane properties, single action potential shape (Table 1), and firing pattern (Figure 1B).

\section{Excitatory connections between ACC neurons}

To study excitatory synaptic transmission in the ACC, we recorded synaptic transmissions between two pyramidal neurons (Py-Py) or from a pyramidal neuron to an interneuron (Py-In). Presynaptic neurons were recorded under current clamp and action potentials were induced by current injection. The postsynaptic neurons were recorded under voltage clamp holding at $-70 \mathrm{mV}$. Successful recordings were obtained in 14 pairs of two pyramidal neurons in layer II/III. When a single action potential was evoked in the presynaptic neuron, monosynaptic inward currents, which are putatively glutamatergic, were obtained in postsynaptic neurons with amplitude of 12.1 $\pm 3.0 \mathrm{pA}$ and a failure rate of $19.0 \pm 6.1 \%(\mathrm{n}=14)$ (Figure $2 \mathrm{~A}$ and $2 \mathrm{C}$ ). When we recorded the pair of pyramidal neuron to interneurons, 13 pairs exhibited glutamatergic responses with of $10.4 \pm 1.3 \mathrm{pA}$ and a failure rate of 31.5 $\pm 8.4 \%(\mathrm{n}=13)$ (Figure $2 \mathrm{~B}$ and $2 \mathrm{C})$. Among them, 4 interneurons were located in layer I and another 9 neu-

Table I: Membrane properties and action potential parameters in pyramidal neurons and interneurons in the superficial layers of the ACC

\section{Pyramidal neuron}

15

$161.5 \pm 5.7$

$206.3 \pm 24.9$

$4.0 \pm 0.2$

$-72.9 \pm 1.7$

$-43.4 \pm 1.2$

$92.0 \pm 1.2$

$1.3 \pm 0.1$
Interneuron

15

$44.2 \pm 3.0$

$297.2 \pm 30.62$

$1.0 \pm 0.1$

$-70.2 \pm 1.5$

$-44.7 \pm 0.7$

$65.1 \pm 2.8$

$0.79 \pm 0.04$
Significant Difference
$P<0.001$

$P<0.05$

$P<0.001$

$P=0.23$

$P=0.33$

$P<0.001$

$P<0.001$ 
A

$$
\text { Pyrmidal } \longrightarrow \text { Pyrmidal }
$$
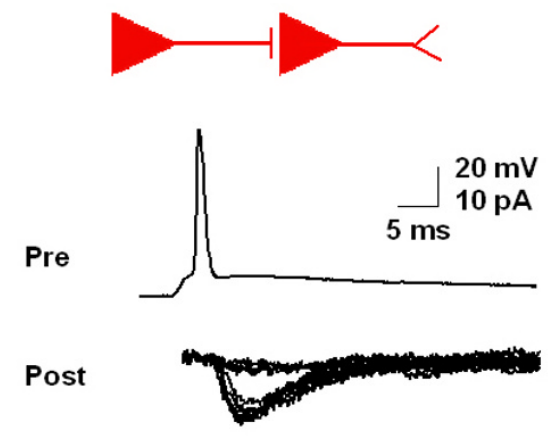

Average

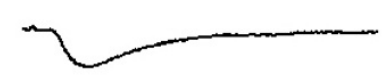

B

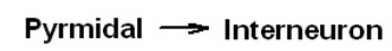

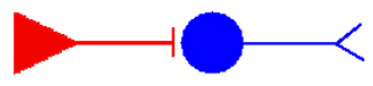

Pre $\underbrace{\frac{10 \mathrm{~mA}}{10 \mathrm{mV}}}_{5 \mathrm{~ms}}$

Post

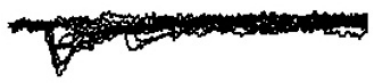

Average

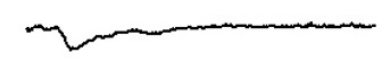

C

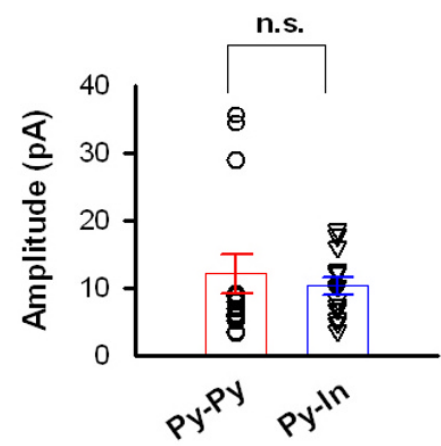

$\mathbf{F}$

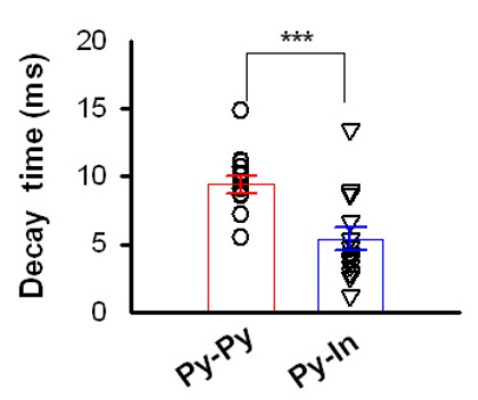

D
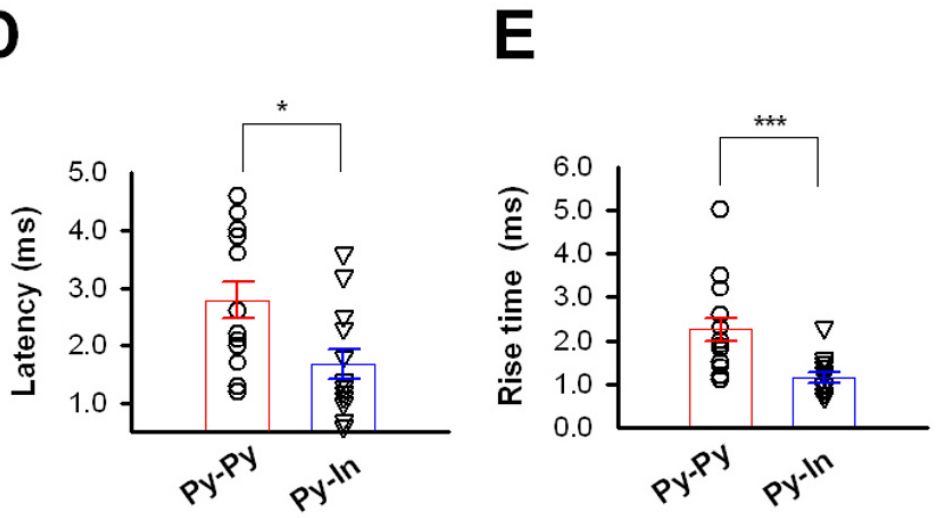

$G$

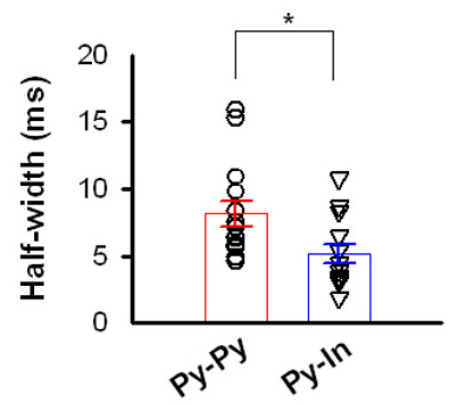

\section{Figure 2}

Excitatory synaptic transmission in the ACC. (A) Diagram and sample traces of synaptic connections from pyramidal neurons to pyramidal neurons. Pre, action potential is induced in the presynaptic pyramidal neuron under current clamp. Post, inward uEPSCs with failure are recorded in the postsynaptic neuron under voltage clamp holding at --70 mV. Average, the trace of the average of 10 original traces. (B) Diagram and sample traces of synaptic connections from pyramidal neurons to interneurons. (C) Pooled data (open circle and triangle) and summarized results (bar graph) showing the amplitude of uEPSCs for Py-Py and Py-In. n.s., no significance. (D-G) Pooled data (open circle and triangle) and summarized results (bar graph) indicate the latency $(D)$, rise time $(E)$, decay time $(F)$ and half-width $(G)$ of uEPSCs for Py-Py and Py-In. Unitary EPSCs from Py-In pairs have faster kinetics than those from Py-Py pairs. $* P<0.05$, $* * P<0.01, * * * P<0.001$. 
rons were located in layer II/III. Since there is no obvious difference uEPSCs between these two groups of interneurons, data were pooled together. These results indicate that excitatory connections exist between pyramidal neurons in layer II/III and pyramidal neuron to interneurons in both layer I and layer II/III.

Next, we compared the latency and kinetics of uEPSCs between pairs of Py-Py and Py-In. We found that the uEPSCs to interneurons (Py-In) have significant shorter latency than those to pyramidal neurons (Py-Py) (Py-Py, $2.8 \pm 0.3, \mathrm{n}=14$; Py-In, $1.7 \pm 0.3, \mathrm{n}=13 ; \mathrm{P}<0.05$ ) (Figure 2D). Moreover, the kinetics of uEPSC to interneurons is dramatically faster than that to pyramidal neurons, showing shorter rise time, decay time and half-width (Figure 2E-G).

\section{Inhibitory connections between ACC neurons}

We then studied inhibitory synaptic transmissions from an interneuron to a pyramidal neuron (In-Py) or between two interneurons (In-In). Presynaptic interneurons were recorded under current clamp while postsynaptic pyramidal neurons or interneurons were under voltage-clamp holding at $0 \mathrm{mV}$. Therefore, the putatively GABAergic outward current were obtained in postsynaptic neurons. We successfully recorded 14 functional pairs of In-Py connections, in which uIPSCs showed amplitude of $56.4 \pm 14.4$ $\mathrm{pA}$ and a failure rate of $3.9 \pm 2.7 \%(\mathrm{n}=14)$ (Figure $3 \mathrm{~A}$ and 3C). Among them, 6 interneurons were recorded in the layer I while the other 8 interneurons were found in layers II/III. For In-In connections, 8 functional pairs were recorded. The amplitude of uIPSCs was $38.8 \pm 20.9 \mathrm{pA}$ and the failure rate was $18.3 \pm 10.7 \%(n=7)$ (Figure $3 \mathrm{~B}$ and 3C). Among 8 functional In-In connections, 2 paired neurons were both in layer I and another 2 paired neurons were both in layer II. In the rest 4 pairs, neurons were in layers I and II/III respectively. These results suggest that interneurons in layers I or II/III could target to both pyramidal neurons and interneurons all over the superficial layers. We also compared the properties of uIPSCs in pairs of In-Py and In-In neuron. Significant faster kinetics, such as rise time, decay time and half-width, were found for uIPSCs in interneurons than those in pyramidal neurons (Figure 3E-G). However, the latency of IPSCs was not statistically significant between two groups $(P=0.09$, Figure 3D).

\section{Reciprocal connections between ACC neurons}

We noticed that in functional pairs of ACC neurons, there exist reciprocal connections. For example, 2 out of 14 pairs of Py-Py show bidirectional transmission (Figure $4 \mathrm{~A}$ ). Between pyramidal neurons and interneurons, there are 8 out of 27 pairs were reciprocal (Figure 4B). Among 8 pairs of In-In, 2 pairs were bidirectional (Figure 4C), including 1 pair of electric-like coupling (Figure 4D). The electrically coupled interneurons were located in layers I and II/III respectively. There is no latency between stimulations and responses. Moreover, action potential firing in one neuron induced immediate inward current with fast kinetics of $<3$ ms half-width (Figure 4D)

\section{Morphology of neurons with functional connections in the ACC}

Biocytin was loaded into some of the recorded neurons, which was followed by immunostaining and imaging of neuronal morphology under confocal microscopy. Under confocal microscopy, all of the identified pyramidal neurons $(n=8)$ and interneurons $(n=6)$ were located in layers II and III of ACC. Pyramidal cells have bigger somata (15-30 $\mu \mathrm{m})$ than interneurons $(10-15 \mu \mathrm{m})$. Typically, a pyramidal cell had one main apical dendrites, which ascended from the soma, stretched into layer I and bifurcated in a tuft there. The proximal dendrites gave off many branches from the soma and arborized extensively, covering a spherical field with diameter equaled to 200-400 $\mu \mathrm{m}$. A typical interneuron has no or short apical dendrite, less branches of proximal dendrites and smaller covering field with diameter equaled to $40-100 \mu \mathrm{m}$. Figure 5 shows pairs of Py-Py (Figure 5A-C) and In-Py (Figure 5D-F) neurons. By using three-dimensional analysis, we found that presynaptic boutons (with obvious varicose swellings) have very close contacts with postsynaptic spines (Figure $5 \mathrm{C}$ and $5 \mathrm{~F}$ ), suggesting the possible locations of synaptic contacts.

\section{Pharmacological identification of excitatory and inhibitory connections between ACC neurons}

To study further the pharmacological properties of the excitatory and inhibitory connections between ACC neurons, the AMPA/kainate receptor antagonist CNQX (10 $\mu \mathrm{M}$ ) and the $\mathrm{GABA}_{\mathrm{A}}$ receptor antagonist picrotoxin (PTX, $50 \mu \mathrm{M}$ ) were used (Figure 6 ). We found that CNQX could completely inhibit the synaptic responses of Py-In pairs ( $\mathrm{n}$ = 3, Figure 6A), while PTX blocked the synaptic responses of In-Py pairs ( $n=3$, Figure $6 \mathrm{~B}$ ) after bath application of each antagonist for 5 minutes. These results confirm the identities of uEPSCs and uIPSCs, which are mediated by $\mathrm{AMPA} /$ kainate and $\mathrm{GABA}_{\mathrm{A}}$ receptors, respectively.

\section{Intracellular CI--dependent IPSCs from interneurons to pyramidal neurons}

During development or under pathological conditions, there is a higher concentration of intracellular $\mathrm{Cl}^{-}\left(\left[\mathrm{Cl}^{-}\right]_{\mathrm{i}}\right)$, which mediates the excitatory, instead of the typically inhibitory, effect of GABAergic responses during those states [24-27]. We wanted to see whether the polarity and properties of GABAergic transmission are also dependent on the postsynaptic $\mathrm{Cl}^{-}$concentration in the ACC. To address this question, we performed dual recordings in interneuron-pyramidal neuron pairs with low $\left[\mathrm{Cl}^{-}\right]_{\mathrm{i}}(7$ 
A

$$
\text { Interneuron } \rightarrow \text { Pyrmidal }
$$
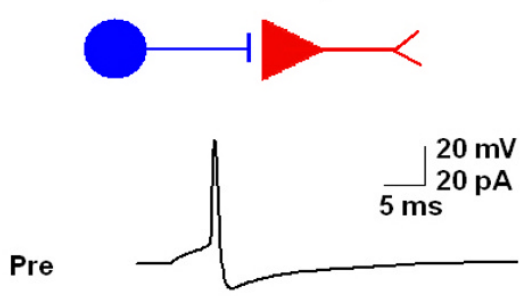

Post

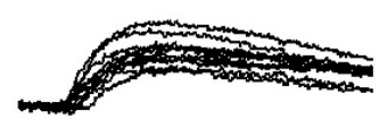

Average

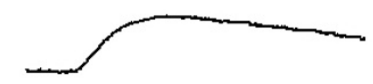

B

$$
\text { Interneuron } \rightarrow \text { Interneuron }
$$
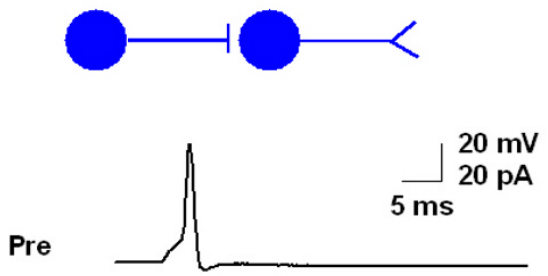

Post

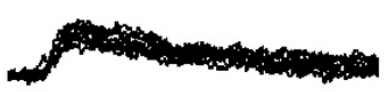

Average

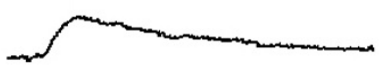

C

D

E
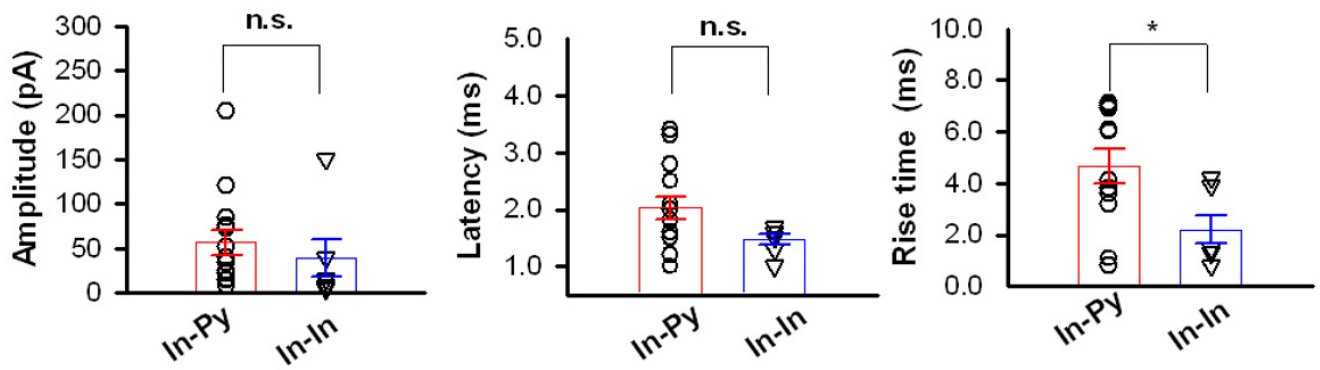

F

G
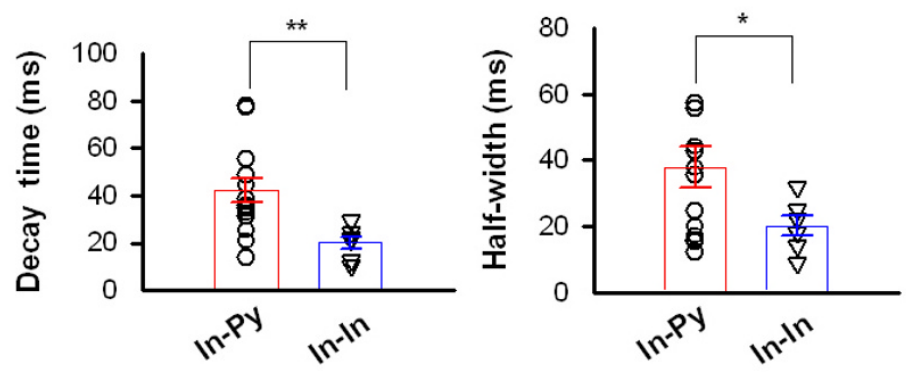

\section{Figure 3}

Inhibitory synaptic transmission in the ACC. $(\boldsymbol{A})$ Diagram and sample traces of synaptic connections from interneurons to pyramidal neurons. Pre, action potential is induced in the presynaptic interneuron under current clamp. Post, outward ulP$\mathrm{SCs}$ is recorded in postsynaptic neuron under voltage clamp holding at $0 \mathrm{mV}$. Average, the trace of the average of 10 original traces. (B) Diagram and sample traces of synaptic connections from interneuron to interneuron. (C) Pooled data (open circle and triangle) and summarized results (bar graph) showing the amplitude of uIPSCs for In-Py and In-In. (D-G) Pooled data (open circle and triangle) and summarized results (bar graph) indicate the latency $(D)$, rise time $(\boldsymbol{E})$, decay time $(\boldsymbol{F})$ and half-width (G) of uEPSCs for In-Py and In-In. Unitary IPSCs from In-In pairs have faster kinetics than those from In-Py pairs. 
A

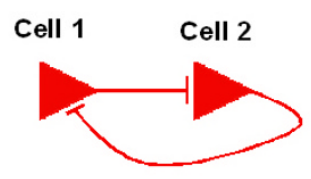

B

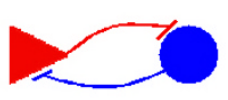

C

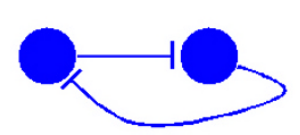

D

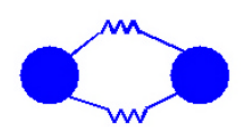

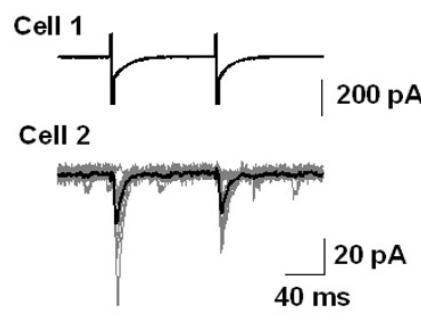
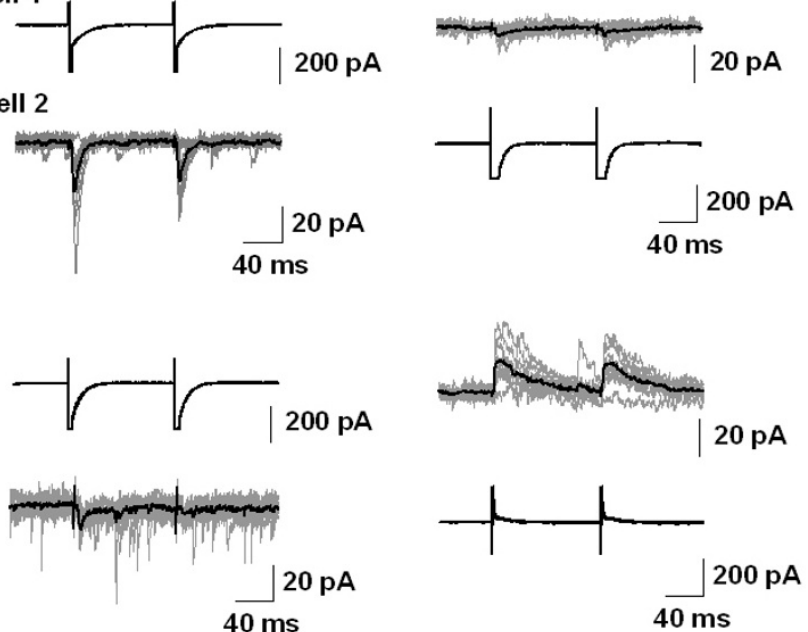

$40 \mathrm{~ms}$
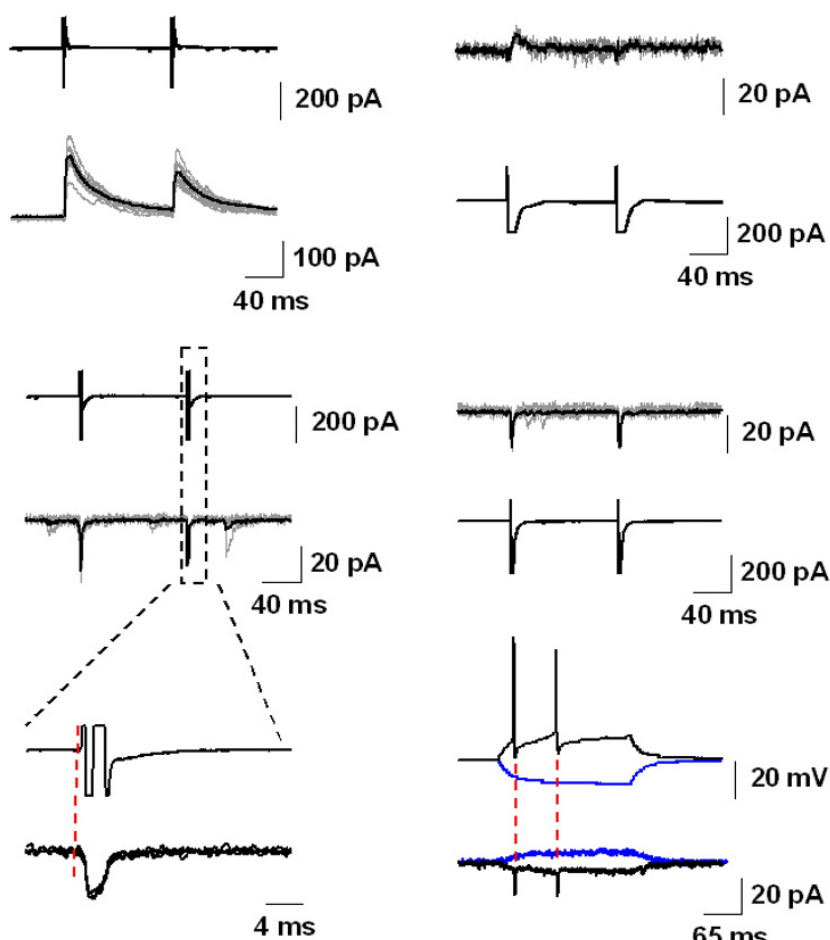
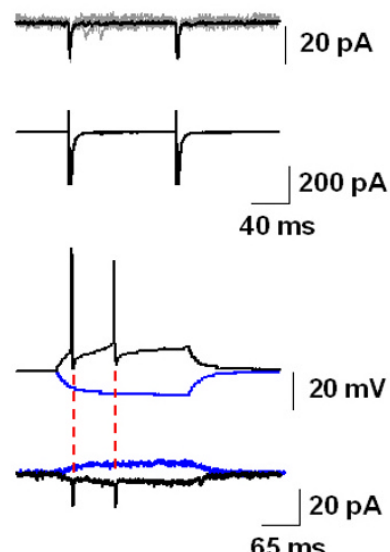

Figure 4

Bidirectional connections between ACC neurons. $(A)$ Reciprocal connections between two pyramidal neurons. Left, diagram of the connection. Middle, two successive stimulations ( $(\mathrm{ms}, 90 \mathrm{mV}$ ) in cell I (upper) induced inward currents in cell 2 (lower). Original traces are shown in gray and the averaged trace is shown in black. Right, stimulations in cell 2 (lower) induced inward currents in cell I (upper). Original traces are shown in grey and the averaged trace is shown in black. (B) Diagram and sample traces of reciprocal connections between pyramidal neurons and interneurons. (C) Diagram and sample traces of reciprocal connections between two interneurons. $(D)$ Diagram and sample traces of electrical coupling between two interneurons. The dashed rectangle is magnified and the latency of stimulation and response is indicated by red dash lines. In the right lower corner, action potentials induced in cell I are coupled with inward currents in cell 2. 

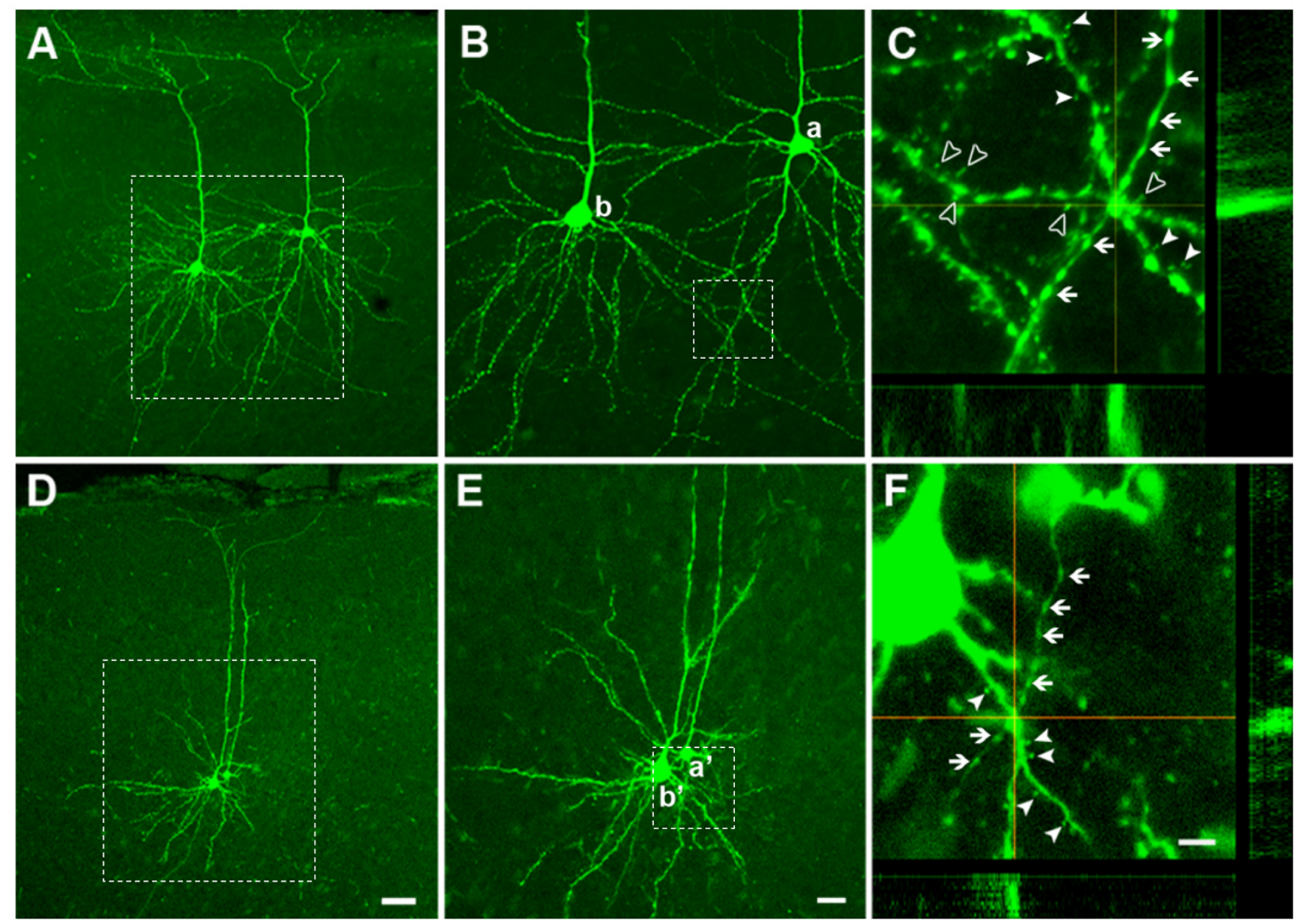

\section{Figure 5}

Confocal images of biocytin-labeled ACC neurons with functional connections. ( $A-C)$ Two pyramidal cells (a and b) show the possible synaptic contacts. The rectangular area in $A$ is shown in $B$ with a higher magnification. $C$ is the three-dimensional view of the rectangular field in $B$, in which an axon from cell (a) makes close connections with two dendrites from cell (b). Arrows show the axon varicose swellings and blank and filled triangles show the dendrite spines. (D-F) One interneuron (a') makes close contact with one pyramidal cell (b'). The rectangular area in $D$ is shown in $E$ with a higher magnification. $F$ is the three-dimensional view of the rectangular area in $\mathrm{E}$, in which an axon from cell (a') makes close connections with one dendrite from cell (b'). Bars equal to 50 ( $A$ and $D), 20$ ( $B$ and $E)$ and 5 (C and F) microns, respectively.

$\mathrm{mM})$ or high $\left[\mathrm{Cl}^{-}\right]_{\mathrm{i}}(67 \mathrm{mM})$. In functional connections from an interneuron to a pyramidal neuron, outward current was evoked at a holding potential of $0 \mathrm{mV}$, while no current was observed at a holding potential of $-70 \mathrm{mV}$ with low $\left[\mathrm{Cl}^{-}\right]_{i}$. In the same neuron, the inward current appeared at a holding potential of $-70 \mathrm{mV}$ after a re-patch of the postsynaptic neuron with high $\left[\mathrm{Cl}^{-}\right]_{\mathrm{i}}$ (Figure $7 \mathrm{~A}$ ). These results confirmed that the polarity of the GABAergic response is determined by the concentration of postsynaptic $\mathrm{Cl}^{-}$. We successfully recorded 19 pairs of In-Py with high $\left[\mathrm{Cl}^{-}\right]_{\mathrm{i}}$ in the postsynaptic pyramidal neurons. The kinetics of outward and inward GABAergic responses were compared and we found the inward GABAergic responses exhibited a much shorter rise time, decay time and halfwidth $(\mathrm{P}<0.001$, Figure $7 \mathrm{~B}-\mathrm{D})$. The different kinetics could be attritubed to the different holding potentials [28] or the polarity of the GABAergic current influenced by the different $\left[\mathrm{Cl}^{-}\right]_{\mathrm{i}}$.

\section{Paired-pulse depression of synaptic connections between ACC neurons}

The paired-pulse ratio (PPR) is a commonly used parameter to indicate the presynaptic release probability [29]. Therefore, we wanted to compare the PPRs in the four types of synaptic connections described above. In contrast to what we know about excitatory transmission in the ACC [30], the PPR for uEPSC at a $100 \mathrm{~ms}$ interval showed significant depression rather than facilitation (for Py-Py, $\mathrm{n}$ $=9$ out of 10 pairs, $\mathrm{P}<0.01$; for Py-In, 9 out of 12 pairs, $\mathrm{P}$ $<0.01$, Figure $8 \mathrm{~A}$ and $8 \mathrm{~B}$ ). There is also a significant 
A

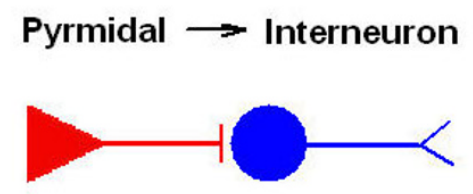

\section{Control}

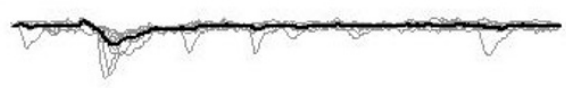

$+10 \mu \mathrm{M} C N Q X$
B
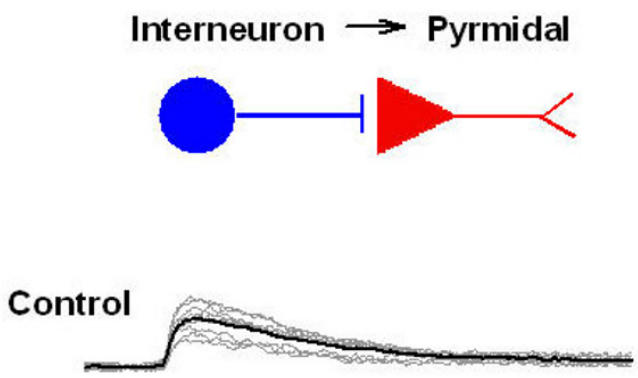

$+50 \mu \mathrm{M}$ PTX

\section{$20 \mathrm{pA}$ \\ $4 \mathrm{~ms}$}

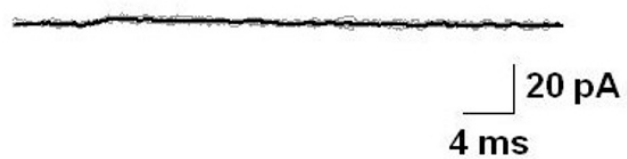

\section{Figure 6}

Pharmacological studies of uEPSCs and uIPSCs. (A) Unitary EPSCs between Py-In were inhibited by CNQX. The postsynaptic interneuron was held at --70 mV. Unitary synaptic responses were obtained by firing presynaptic pyramidal neuron. And the responses were completely abolished by bath application of CNQX. Original traces were shown in grey and the averaged trace was shown in black. (B) Unitary IPSCs between In-Py were blocked by PTX. Postsynaptic pyramidal neuron was holding at $0 \mathrm{mV}$. Unitary synaptic responses were obtained by firing presynaptic interneuron. And the responses were completely abolished by bath application of PTX. Original traces were shown in grey and the averaged trace was shown in black.

paired-pulse depression of uIPSCs for pairs of In-Py and In-In, which is consistent with the PPR of evoked IPSCs by bulk local stimulation [22]. No difference was found for the PPR between the excitatory and inhibitory synaptic transmission (Figure 8B).

When we calculated the PPR, we ruled out the event if the first stimulation failed to induce the current. Therefore, we compared the failure rate of the four types of synaptic transmission. Noticeably, there is very low failure in the pair of In-Py, suggesting the high release probabilities of this type of synaptic connection. When we pooled the excitatory and inhibitory transmission respectively, we found that the failure rate is higher in excitatory transmission than that in inhibitory synaptic transmission (Figure $8 \mathrm{C})$.

\section{Discussion}

In the present study, we examined the intracortical connections of pyramidal neurons and/or interneurons in the superficial layers of the ACC using dual patch clamp recordings. One technical advantage of dual recording is that it avoids stimulation of passing fibers and allows investigation of local synaptic connections. Four types of synaptic pairs were characterized: (1) from pyramidal neuron to pyramidal neuron; (2) from pyramidal neuron to interneuron; (3) from interneuron to pyramidal neuron; (4) from interneuron to interneuron. In addition, there are reciprocal connections between these pairs. Interestingly, we found that the unitary postsynaptic current showed faster kinetics to interneurons than that to pyramidal neurons. However, the failure rate is higher in glutamatergic transmission than in GABAergic transmission. These results suggest that the postsynaptic neuronal type determines the kinetics of synaptic current, while the presynaptic neuronal type determines the release probability. It has been reported that different AMPA receptors are expressed in pyramidal neurons and interneurons [31]. For example, a GluR2-lacking AMPA receptor is expressed mainly in interneurons but not in pyramidal neurons in the amygdala [32]. Therefore, this may also explain the different kinetics of uEPSCs in pyramidal neurons and interneurons in the anterior cingulate cortex. Similarly, different subunit composition of $\mathrm{GABA}_{\mathrm{A}}$ receptors may also underlie the different kinetics of uIPSCs in pyramidal neurons and interneurons. Future experiments are needed to address the questions. 


\section{A}

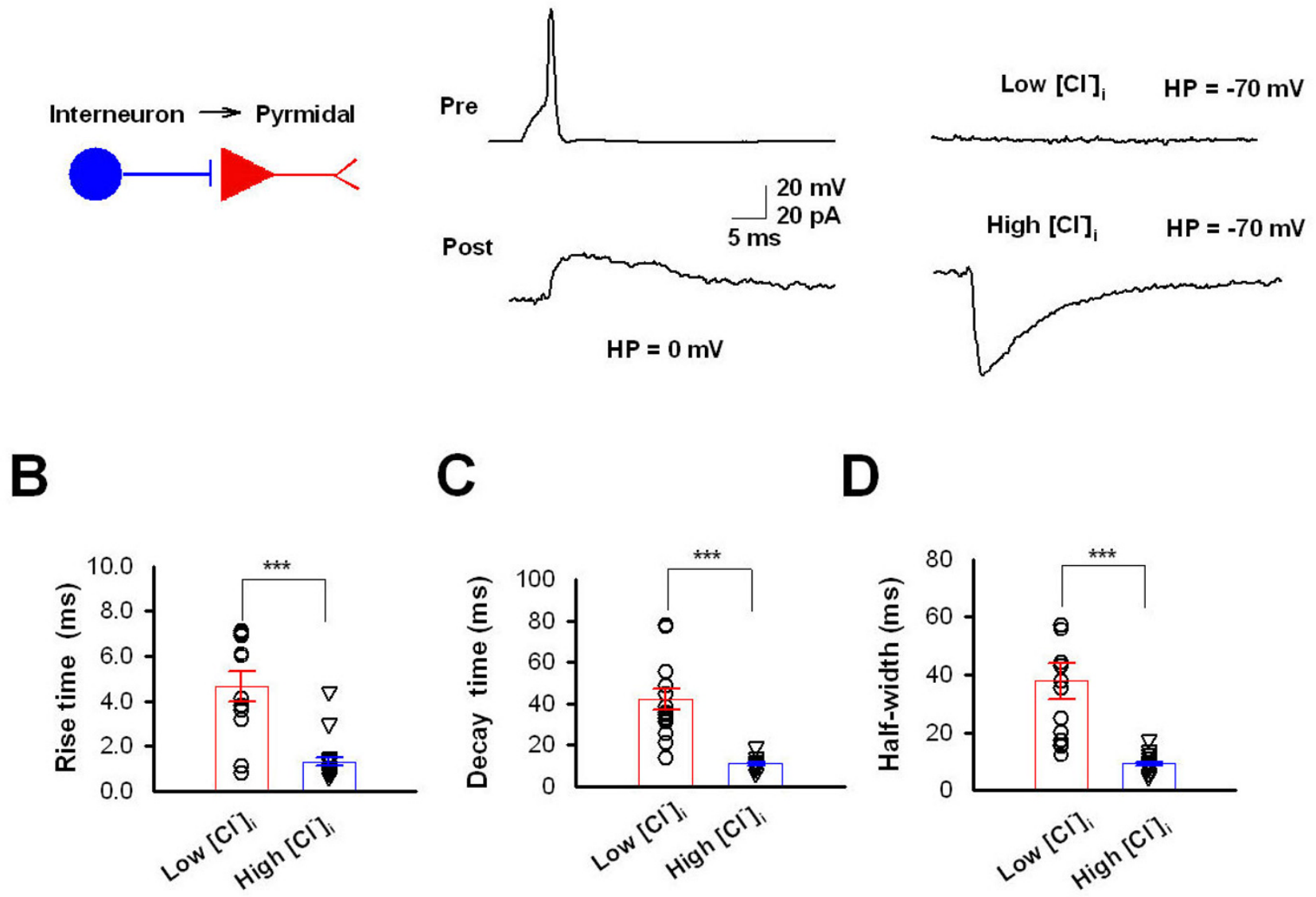

\section{Figure 7}

Unitary IPSCs in ACC pyramidal neuron with high $\left[\mathrm{Cl}^{-}\right]_{\mathrm{i}} \cdot(\boldsymbol{A})$ Outward and inward ulPSCs in ACC pyramidal neurons with low $\left[\mathrm{Cl}^{-}\right]_{\mathrm{i}}$ and high $\left[\mathrm{Cl}_{\mathrm{i}}\right.$, respectively. Left, diagram of the connection. Middle, interneuronal firing (upper) induced outward uIPSCs in the postsynaptic pyramidal neurons at a holding potential of $0 \mathrm{mV}$ with low [Cl- $]_{\mathrm{i}}$ (lower). Right, no current is induced if holding at of --70 mV with low $\mathrm{CCl}_{\mathrm{i}}$ (upper), while an inward current is obtained at a holding potential of --70 $\mathrm{mV}$ if the same neuron is re-patched with high $\left[\mathrm{Cl}^{-}\right]_{i}$ intracellular solution (upper). (B-D) Pooled data (open circle and triangle) and summarized results (bar graph) indicate the rise time (B), decay time (C) and half-width (D) of ulPSCs obtained under low [Cl]$_{i}$ and high $\left[\mathrm{Cl}^{-}\right]_{i}$. Unitary IPSCs with high $\left[\mathrm{Cl}^{-}\right]_{i}$ have faster kinetics than those with low $\left[\mathrm{Cl}^{-}\right]_{i}$.

We have demonstrated the existence in the ACC of all proposed connections shown in Figure 1. For example, pyramidal neurons form synapses with pyramidal neurons and interneurons in layers II/III and also send projections to layer I interneurons; interneurons in layer I can target pyramidal neurons and interneurons in layers II/III, and also interneurons in layer I. In addition, interneurons in layers II/III can form synapses with all types of cells in the same layer or those in layer I. Since the pyramidal neurons and interneurons may also be divided into several subtypes based on their firing patterns [24,33], the present study underestimates the complexity of synaptic connections. The synaptic properties of ACC neurons need to be characterized further based on the different subtypes of pyramidal neurons and interneurons, rather than just by comparing the excitatory and inhibitory synaptic transmission or connections between pyramidal neurons and interneurons.

Short-term plasticity such as paired-pulse facilitation (PPF) and depression (PPD) is important for synaptic communication in the brain. PPF is generally explained as an increase of release probability during a second stimulus, arising from prior accumulation of residual $\mathrm{Ca}^{2+}$ near 

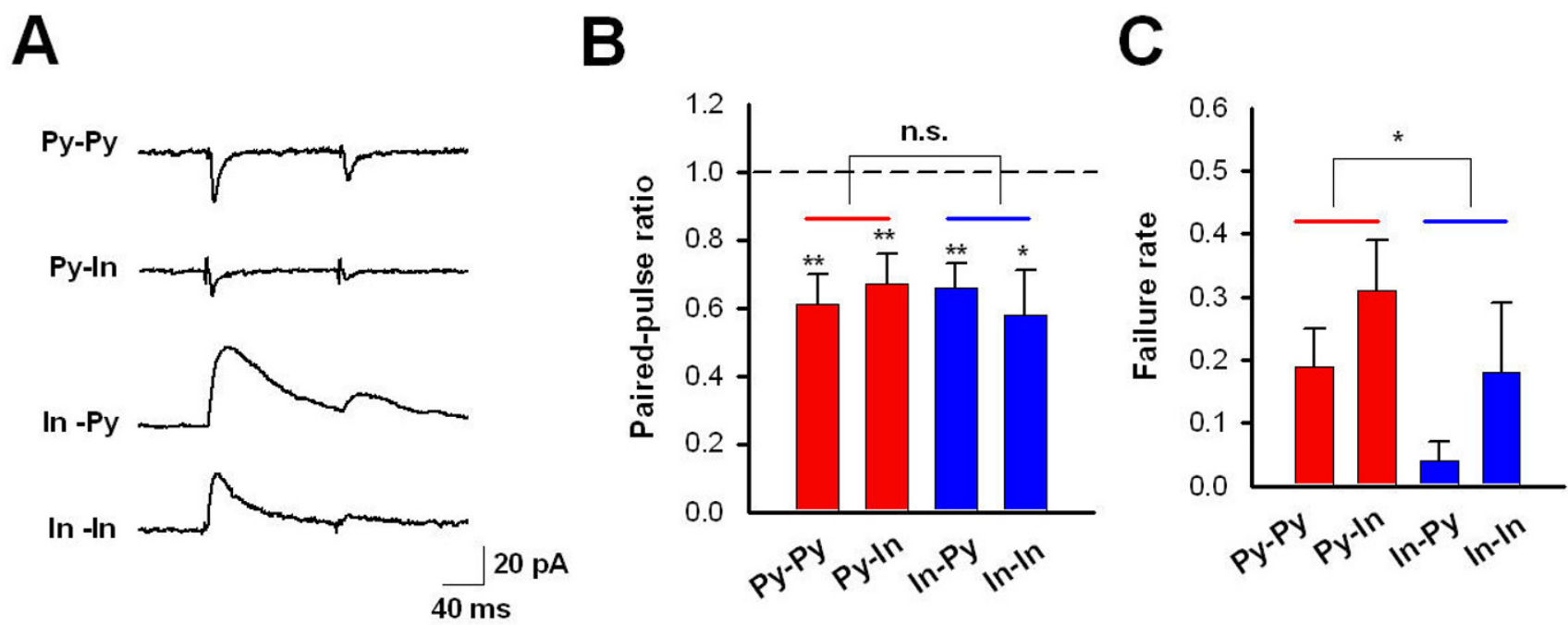

\section{Figure 8}

Paired-pulse ratio and failure rate of four types of synapses in the ACC. (A) Traces showing postsynaptic currents in the four types of synaptic connections induced by the two successive presynaptic stimulations (I00 ms interval). (B) Summarized results of paired-pulse depression in all four types of synaptic connections. There is no significant difference between excitatory (pooled Py-Py and Py-In, indicated as a red line) and inhibitory (pooled In-Py and In-In, indicated as a blue line) synaptic transmission. (C) Summarized results of failure rates in all four types of synaptic connections. There is a significantly lower failure rate for inhibitory (pooled In-Py and In-In, indicated as blue line) than for excitatory (pooled Py-Py and Py-In, indicated as red line) transmission.

active zones, while PPD is thought to reflect depletion of the pool of readily releasable vesicles or inhibition of calcium currents in the presynaptic terminal $[29,34]$. In the present study, we found that uIPSCs exhibit PPD, which is in agreement with our previous work using field stimulation [22] and suggest the possible high release probabilities of GABAergic synapses in the ACC. Consistently, the failure rate of inhibitory transmission is low, particularly for the pair of In-Py. However, we found that uEPSCs also show PPD in most pairs recorded, which is in contrast to the PPF of evoked EPSCs induced by field stimulations in the ACC $[30,35]$. Two reasons may account for the discrepancy. First, there are differences in the stimulating fibers. We only activate the presynaptic pyramidal neurons in the layer II/III in the current study, while the bulk stimulation in the layer II/III could also trigger the release from synaptic terminals originating from the medial thalamus. Strong PPF was reported for synaptic transmission from medial thalamus to layer II/III in the ACC [36]. Second, when we analyze the paired-pulse ratio, we exclude events with failure at the first stimulation. Considering around $20-30 \%$ failure rate of uEPSCs and the most likely PPF of these events, the ratio may not reflect the short- term plasticity in situ. It has also been reported that uEPSC to interneurons show different short-term plasticity dependent on postsynaptic interneuron types, such as PPD for fast-spiking neurons or multipolar cells while PPF for low-threshold spiking neuron or bitufted cells [37-39]. Although we did not identify the interneuronal types in the present study, we believe that most interneurons we recorded are fast-spiking interneurons based on the firing patterns (Figure 1B) and action potential properties (Table 1).

Neurons in layers $\mathrm{V}$ and VI provide the main output of the ACC. The communication between these layers and superficial layers is critical for the integration of the ACC circuit and the execution its related brain functions [40]. Therefore, future study is needed to extend the characterization of synaptic connections between deep and superficial layers in the ACC. In addition, in vitro and in vivo studies have shown plastic changes in the ACC after pathological conditions such as chronic pain [14-16,40,41]. Future experiments using dual recording could explore and uncover the synaptic mechanisms of these plastic changes at the single synapse level. 


\section{Competing interests}

The authors declare that they have no competing interests.

\section{Authors' contributions}

LJW, XL and MR carried out electrophysiological experiments, TC performed staining experiments, LJW drafted the manuscript, $\mathrm{MZ}$ coordinated the study. All authors read and approved the final manuscript.

\section{Acknowledgements}

Supported by grants from the Canadian Institutes of Health Research (CIHR81086, CIHR84256), the EJLB-CIHR Michael Smith Chair in Neurosciences and Mental Health, and the Canada Research Chair to M. Z. M.Z. is also supported by the WCU project at Seoul National University. L.-J.W. is supported by postdoctoral fellowships from the Canadian Institutes of Health Research and Fragile $\times$ Research Foundation of Canada. We thank Martin $\vee$ Kurtev for critical reading of the manuscript.

\section{References}

I. Leknes S, Tracey I: A common neurobiology for pain and pleasure. Nat Rev Neurosci 2008, 9:3 I4-320.

2. Frankland PW, Bontempi B, Talton LE, Kaczmarek L, Silva AJ: The involvement of the anterior cingulate cortex in remote contextual fear memory. Science 2004, 304:88I-883.

3. Maviel T, Durkin TP, Menzaghi F, Bontempi B: Sites of neocortical reorganization critical for remote spatial memory. Science 2004, 305:96-99.

4. Zhuo M: Cortical excitation and chronic pain. Trends Neurosci 2008, 31:199-207.

5. Devinsky O, Morrell MJ, Vogt BA: Contributions of anterior cingulate cortex to behaviour. Brain 1995, I I 8(Pt I):279-306.

6. Zhuo M: Neuronal mechanism for neuropathic pain. Mol Pain 2007, 3: 14.

7. Zhuo M: A synaptic model for pain: long-term potentiation in the anterior cingulate cortex. Mol Cells 2007, 23:259-27I.

8. Neugebauer V, Galhardo V, Maione S, Mackey SC: Forebrain pain mechanisms. Brain Res Rev 2009, 60:226-242.

9. Wu LJ, Zhuo M: Targeting the NMDA Receptor Subunit NR2B for the Treatment of Neuropathic Pain. Neurotherapeutics 2009, 6:693-702.

10. Calejesan AA, Kim SJ, Zhuo M: Descending facilitatory modulation of a behavioral nociceptive response by stimulation in the adult rat anterior cingulate cortex. Eur J Pain 2000, 4:83-96.

II. Tang J, Ko S, Ding HK, Qiu CS, Calejesan AA, Zhuo M: Pavlovian fear memory induced by activation in the anterior cingulate cortex. Mol Pain 2005, 1:6.

12. Johansen JP, Fields HL: Glutamatergic activation of anterior cingulate cortex produces an aversive teaching signal. Nat Neurosci 2004, 7:398-403.

13. Zhuo M: Plasticity of NMDA receptor NR2B subunit in memory and chronic pain. Mol Brain 2009, 2:4.

14. Wu LJ, Toyoda H, Zhao MG, Lee YS, Tang J, Ko SW, Jia YH, Shum FW, Zerbinatti CV, Bu G, et al.: Upregulation of forebrain NMDA NR2B receptors contributes to behavioral sensitization after inflammation. J Neurosci 2005, 25: I I 107- I I I I6.

15. Wei F, Li P, Zhuo M: Loss of synaptic depression in mammalian anterior cingulate cortex after amputation. J Neurosci 1999 , 19:9346-9354

16. Wei F, Zhuo M: Potentiation of sensory responses in the anterior cingulate cortex following digit amputation in the anaesthetised rat. J Physiol 200I, 532:823-833.

17. Morgane PJ, Galler JR, Mokler DJ: A review of systems and networks of the limbic forebrain/limbic midbrain. Prog Neurobiol 2005, 75: 143-160.

18. Wang CC, Shyu BC: Differential projections from the mediodorsal and centrolateral thalamic nuclei to the frontal cortex in rats. Brain Res 2004, 995:226-235.

19. Shibata $H$ : Efferent projections from the anterior thalamic nuclei to the cingulate cortex in the rat. J Comp Neurol 1993, 330:533-542.
20. Gabbott PL, Warner TA, Jays PR, Bacon S): Areal and synaptic interconnectivity of prelimbic (area 32), infralimbic (area 25) and insular cortices in the rat. Brain Res 2003, 993:59-71.

2I. Wu LJ, Zhao MG, Toyoda H, Ko SW, Zhuo M: Kainate receptormediated synaptic transmission in the adult anterior cingulate cortex. J Neurophysiol 2005, 94: 1805-18।3.

22. Wu LJ, Xu H, Ren M, Zhuo M: Genetic and pharmacological studies of GluR5 modulation of inhibitory synaptic transmission in the anterior cingulate cortex of adult mice. Dev Neurobiol 2007, 67: 146-157.

23. Wu LJ, Ko SW, Toyoda H, Zhao MG, Xu H, Vadakkan KI, Ren M, Knifed E, Shum F, Quan J, et al:: Increased anxiety-like behavior and enhanced synaptic efficacy in the amygdala of GluR5 knockout mice. PLOS ONE 2007, 2:el 67.

24. Wu LJ, Kim SS, Li X, Zhang F, Zhuo M: Sexual attraction enhances glutamate transmission in mammalian anterior cingulate cortex. Mol Brain 2009, 2:9.

25. Rivera C, Voipio J, Payne JA, Ruusuvuori E, Lahtinen H, Lamsa K, Pirvola U, Saarma M, Kaila K: The K+/Cl- co-transporter $\mathrm{KCC2}$ renders GABA hyperpolarizing during neuronal maturation. Nature 1999, 397:25I-255.

26. Nabekura J, Ueno T, Okabe A, Furuta A, Iwaki T, Shimizu-Okabe C, Fukuda $A$, Akaike N: Reduction of KCC2 expression and GABAA receptor-mediated excitation after in vivo axonal injury. J Neurosci 2002, 22:44I2-44I7.

27. Coull JA, Boudreau D, Bachand K, Prescott SA, Nault F, Sik A, De Koninck $P$, De Koninck $Y$ : Trans-synaptic shift in anion gradient in spinal lamina I neurons as a mechanism of neuropathic pain. Nature 2003, 424:938-942.

28. Oh DJ, Dichter MA: Desensitization of GABA-induced currents in cultured rat hippocampal neurons. Neuroscience 1992, 49:57I-576.

29. Zucker RS, Regehr WG: Short-term synaptic plasticity. Annu Rev Physiol 2002, 64:355-405.

30. Wu LJ, Steenland HW, Kim SS, Isiegas C, Abel T, Kaang BK, Zhuo M: Enhancement of presynaptic glutamate release and persistent inflammatory pain by increasing neuronal CAMP in the anterior cingulate cortex. Mol Pain 2008, 4:40.

31. Hestrin S: Different glutamate receptor channels mediate fast excitatory synaptic currents in inhibitory and excitatory cortical neurons. Neuron 1993, I1:1083-1091.

32. Mahanty NK, Sah P: Calcium-permeable AMPA receptors mediate long-term potentiation in interneurons in the amygdala. Nature 1998, 394:683-687.

33. Bean BP: The action potential in mammalian central neurons. Nat Rev Neurosci 2007, 8:45I-465.

34. Schneggenburger R, Sakaba T, Neher E: Vesicle pools and shortterm synaptic depression: lessons from a large synapse. Trends Neurosci 2002, 25:206-2I2.

35. Zhao MG, Ko SW, Wu LJ, Toyoda H, Xu H, Quan J, Li J, jia Y, Ren M, $\mathrm{Xu} Z \mathrm{ZC}$, et al.: Enhanced presynaptic neurotransmitter release in the anterior cingulate cortex of mice with chronic pain. J Neurosci 2006, 26:8923-8930.

36. Lee CM, Chang WC, Chang KB, Shyu BC: Synaptic organization and input-specific short-term plasticity in anterior cingulate cortical neurons with intact thalamic inputs. Eur J Neurosci 2007, 25:2847-286I.

37. Koester HJ, Johnston D: Target cell-dependent normalization of transmitter release at neocortical synapses. Science 2005 , 308:863-866.

38. Reyes A, Lujan R, Rozov A, Burnashev N, Somogyi P, Sakmann B: Target-cell-specific facilitation and depression in neocortical circuits. Nat Neurosci 1998, I:279-285.

39. Lu JT, Li CY, Zhao JP, Poo MM, Zhang XH: Spike-timing-dependent plasticity of neocortical excitatory synapses on inhibitory interneurons depends on target cell type. J Neurosci 2007, 27:97| I-9720.

40. Gabbott PL, Warner TA, Jays PR, Salway P, Busby SJ: Prefrontal cortex in the rat: projections to subcortical autonomic, motor, and limbic centers. I Comp Neurol 2005, 492: I45-I77.

4I. Xu H, Wu LJ, Wang H, Zhang X, Vadakkan KI, Kim SS, Steenland HW, Zhuo M: Presynaptic and postsynaptic amplifications of neuropathic pain in the anterior cingulate cortex. J Neurosci 2008, 28:7445-7453. 